\section{Complete Genome Sequence of the Fire Blight Pathogen Strain Erwinia amylovora Ea1189}

\author{
Menghao Yu, ${ }^{1}$ Jugpreet Singh, ${ }^{2}$ Awais $\mathrm{Khan}^{2}$ George W. Sundin, ${ }^{3}$ and Youfu Zhao ${ }^{1, \dagger}$ \\ ${ }^{1}$ Department of Crop Sciences, University of Illinois at Urbana-Champaign, IL 61801, U.S.A. \\ 2 Plant Pathology and Plant-Microbe Biology Section, Cornell University, Geneva, NY 14456, U.S.A. \\ ${ }^{3}$ Department of Plant, Soil and Microbial Sciences, Michigan State University, East Lansing, MI 48824, \\ U.S.A.
}

\begin{abstract}
Erwinia amylovora causes fire blight, the most devastating bacterial disease of apples and pears in the United States and worldwide. The model strain E. amylovora Ea1189 has been extensively used to understand bacterial pathogenesis and molecular mechanisms of bacterial-plant interactions. In this work, we sequenced and assembled the de novo genome of Ea1189, using a combination of long Oxford Nanopore Technologies and short Illumina sequence reads. A complete gapless genome assembly of Ea1189 consists of a 3,797,741bp circular chromosome and a 28,259-bp plasmid with 3,472 predicted genes, including 78 transfer RNAs, 22 ribosomal RNAs, and 20 noncoding RNAs. A comparison of the Ea1189 genome to previously sequenced $E$. amylovora complete genomes showed 99.94 to $99.97 \%$ sequence similarity with 314 to 946 single nucleotide polymorphisms. We believe that the availability of the complete genome sequence of strain Ea1189 will further support studies to understand evolution, diversity and structural variations of Erwinia strains, as well as the molecular basis of $E$. amylovora pathogenesis and its interactions with host plants, thus facilitating the development of effective management strategies for this important disease.
\end{abstract}

\section{Genome Announcement}

Fire blight was first described more than 250 years ago and is the first plant disease discovered to be caused by a bacterium (Smits et al. 2017). It is undoubtedly one of the most serious and perplexing bacterial diseases on pomaceous fruit trees, including pear, apple, quince, and several other members of the family Rosaceae (van der Zwet 1979). The causal agent Erwinia amylovora is a gram-negative bacterium, belonging to the Enterobacteriaceae family, which also includes many important human and animal pathogens (Wang et al. 2010). Currently, 135 assemblies of $E$. amylovora at different levels are publicly available in GenBank, including eight with complete genome sequences, three at the chromosome level, 99 at the scaffold level, and 25 at the contig level (National Center for Biotechnology Information [NCBI] Erwinia amylovora database). Previous comparative genomic analyses confirmed a low level of genetic diversity between $E$. amylovora genomes from commercial orchards (Parcey et al. 2020; Singh and Khan 2019; Smits et al. 2010). Genome sequences

\footnotetext{
${ }^{\dagger}$ Corresponding author: Y. Zhao; zhao888@illinois.edu

*The $e$-Xtra logo stands for "electronic extra" and indicates there are supplementary materials published online.
}

The author(s) declare no conflict of interest.

Accepted for publication 17 August 2020.

\footnotetext{
This article is in the public domain and not copyrightable. It may be freely reprinted with customary crediting of the source. The American Phytopathological Society, 2020.
}

\title{
$e-\mathrm{Xtra}^{*}$
}

Funding

This project was supported by the United States Department of Agriculture (USDA) National Institute of Food and Agriculture Agriculture and Food Research Initiative Competitive Grants Program grant number 2016-67013-24812 from the, USDA-Hatch Project ILLU-802-913

(Y. Zhao) and USDA-Specialty Crop Research Initiative grant number 2020-51181-32158. The funders had no role in study design, data collection and analysis, decision to publish, or preparation of the manuscript.

\section{Keywords}

genome, plant-microbe interactions, pathogenesis, Erwinia amylovora 
with relatively low integrity are generally sufficient for taxonomical analysis (Smits 2019). However, to fully understand the diversity of virulence among these highly conserved E. amylovora strains, complete genome assemblies with fewer contigs and improved sequencing quality are essential (Smits 2019; Wang et al. 2010; Zeng et al. 2018). In this study, we report the complete genome sequence of strain E. amylovora Ea1189, which has been extensively used as a model strain in understanding bacterial pathogenesis and plantmicrobe interactions in the past decades (Burse et al. 2004; Khan et al. 2012; Yang et al. 2014; Zhao 2014; Zhao and Qi 2011; Zhao et al. 2005).

E. amylovora Ea1189 was originally isolated from pear (Pyrus communis) in Göttingen, Germany in 1989 (Burse 2003; Burse et al. 2004). After we obtained the isolate, the isolate was stored at $-80^{\circ} \mathrm{C}$ in $15 \%$ glycerol. A single colony was picked from a Luria-Bertani (LB) agar plate for overnight culture in LB broth at $28^{\circ} \mathrm{C}$. Genomic DNA was extracted from overnight bacterial cells using MasterPure complete DNA and RNA purification kit (Epicenter, Madison, WI, U.S.A.). A Nanopore library was prepared using the Rapid Barcoding SQKRBK004 library kit. The library was sequenced with a SpotON R9.4.1 RevC FLO-MIN106 flowcell for a 48-h run on a GridION $\times 5$ sequencer (Oxford Nanopore Technologies (ONT), Oxford). FAST5 files containing raw Nanopore signal data were base-called using Guppy 2.0.5 and were converted to FASTQ format using Guppy 2.3.7 (Wick et al. 2019). The program Porechop 0.2.3 was used to demultiplex the sequences and to trim sequencing adapters. In total, 53,503 reads representing $8.93 \times 10^{8}$ bases were generated. A following quality filtering was conducted to retain $>3-\mathrm{kb}$ longer sequences with quality scores higher than 10, using BBMap 38.36 (Bushnell 2014). A final set of high-quality 46,761 ONT long reads with a total of $8.51 \times 10^{8}$ bases and approximately $218 \times$ coverage of the $E$. amylovora genome was assembled along with previously sequenced Illumina data (Singh and Khan 2019), using the Unicycler hybrid assembly pipeline 0.4.8 (Wick et al. 2017). Canu assembly pipeline 1.9 (Koren et al. 2017) with merely long ONT reads as input was also used to manually confirm Unicycler output. The resulting assembly consisted of two circular contigs of 28,259 bp and 3,797,982 bp in length, which corresponded to the plasmid and chromosome sequences of Ea1189, respectively.

Due to the higher chances of sequence errors in long reads from ONT, we again used the Illumina short read sequences of Ea1189 previously generated by Singh and Khan (2019) to further improve the chromosome and plasmid assemblies of Ea1189. The Illumina data consisted of 303,997 paired-end 250 -bp reads with $4.56 \times 10^{7}$ bases. The quality of the raw sequence reads was assessed using FastQC 0.11.8 (Andrews 2010), and Trimmomatic 0.38 (Bolger et al. 2014) was used to trim reads with a quality score $<20$ over a 4-bp sliding window. After trimming, the read sequences of less than $50 \mathrm{bp}$ length were also eliminated. The remaining high-quality 272,471 paired-end reads with $4.13 \times 10^{7}$ bases were mapped to the Ea1189 assembly with Burrows-Wheeler Aligner 0.7.17 (Li and Durbin 2009) for error correction using Pilon 1.23 (Walker et al. 2014). We ran four iterations of Pilon until no further changes could be integrated. In total, 188 single nucleotide polymorphisms (SNPs), 87 small insertions (totaling 118 bases), and 33 small deletions (totaling 59 bases) were corrected in the improved Ea1189 assembly.

The gapless improved assembly consists of one 28,259-bp plasmid and one 3,797,741bp circular chromosome, with $\mathrm{G}+\mathrm{C}$ content at $50.24 \%$ and $53.61 \%$, respectively. Annotation with the Prokaryotic Genome Annotation Pipeline (Tatusova et al. 2016) yielded a total of 3,472 genes, including 78 transfer RNA, 22 ribosomal RNA, and 20 noncoding RNA genes. To assess the similarity between Ea1189 and other E. amylovora strains, eight complete E. amylovora genome sequences (Supplementary Table S1) were downloaded from the NCBI database and were compared using MUMmer 4.0.0 (Kurtz et al. 2004). The genome similarities between Ea1189 and the selected eight strains range from 99.94 to $99.97 \%$ (Supplementary Table S2), confirming the low diversity of E. amylovora genomes. On the other hand, the number of SNPs between different comparisons with Ea1189 ranged from 314 to 946 (Supplementary Table S2). Regardless of different isolate hosts, there were fewer SNPs among the three European strains (Ea1189, CFBP 1430, and E-2) and among the five Korean strains (FB-86, FB-207, TS3238, FB-307, FB-20), suggesting that geographical origin rather than host of the strains played an important role in SNP variations across these E. amylovora strains. The nontransmissible plasmid pEA29 has been found in most E. amylovora strains, which appears to increase bacterial fitness after colonizing young host tissues (Parcey et al. 2020). A comparative analysis of plasmids from the nine complete

1278 / Molecular Plant-Microbe Interactions 
E. amylovora genomes (Supplementary Tables S1 and S3) confirmed the ubiquity of pEA29 in E. amylovora genomes. The pEA72 plasmid is present only in strain ATCC 4996 and was not detected in Ea1189. Moreover, the pEA29 sequences across the nine strains showed $>99 \%$ identity. Consistent with genome-level SNP variations, pEA29 plasmids in strains from similar geographical locations have almost identical sequences with few SNPs.

The chromosome and plasmid assemblies have been deposited in GenBank under the accession numbers CP055227 and CP055228. The complete genome of Ea1189 is the first E. amylovora genome compiled using ONT and Illumina sequencing technologies. Hybrid assembly of long and continuous ONT reads and short Illumina reads produced a complete genome of the model strain E. amylovora Ea1189 with higher integrity and fewer sequencing errors. Additional complete genome assemblies of $E$. amylovora strains can facilitate a thorough investigation of nucleotide and structural variation across highly conserved $E$. amylovora strains, which could help further our understanding of $E$. amylovora pathogenesis and aid in the design of effective and sustainable management strategies for fire blight control.

\section{Author-Recommended Internet Resources}

NCBI Erwinia amylovora database: https://www.ncbi.nlm.nih.gov/genome/genomes/1159

Porechop 0.2.3: https://github.com/rrwick/Porechop

\section{Literature Cited}

Andrews, S. 2010. FastQC: A quality control tool for high throughput sequence data. Babraham Bioinformatics. Babraham Institute, Cambridge.

Bolger, A. M., Lohse, M., and Usadel, B. 2014. Trimmomatic: A flexible trimmer for Illumina sequence data. Bioinformatics 30:2114-2120.

Burse, A. 2003. Charakterisierung der multidrug-efflux-transporter NorM und AcrAB in Erwinia amylovora. Ph.D. dissertation. University of Marburg, Germany. https://core.ac.uk/download/pdf/144296559.pdf.

Burse, A., Weingart, H., and Ullrich, M. S. 2004. The phytoalexin-inducible multidrug efflux pump AcrAB contributes to virulence in the fire blight pathogen, Erwinia amylovora. Mol. Plant-Microbe Interact 17:43-54.

Bushnell, B. 2014. BBMap: A fast, accurate, splice-aware aligner. Lawrence Berkeley National Lab. LBNL, Berkeley, CA.

Khan, M. A., Zhao, Y. F., and Korban, S. S. 2012. Molecular mechanisms of pathogenesis and resistance to the bacterial pathogen Erwinia amylovora, causal agent of fire blight disease in Rosaceae. Plant Mol. Biol. Report. 30: 247-260.

Koren, S., Walenz, B. P., Berlin, K., Miller, J. R., Bergman, N. H., and Phillippy, A. M. 2017. Canu: Scalable and accurate long-read assembly via adaptive $k$-mer weighting and repeat separation. Genome Res. 27:722-736.

Kurtz, S., Phillippy, A., Delcher, A. L., Smoot, M., Shumway, M., Antonescu, C., and Salzberg, S. L. 2004. Versatile and open software for comparing large genomes. Genome Biol. 5:R12.

Li, H., and Durbin, R. 2009. Fast and accurate short read alignment with BurrowsWheeler transform. Bioinformatics 25:1754-1760.

Parcey, M., Gayder, S., Morley-Senkler, V., Bakkeren, G., Úrbez-Torres, J. R., Ali, S., Castle, A. J., and Svircev, A. M. 2020. Comparative genomic analysis of Erwinia amylovora reveals novel insights in phylogenetic arrangement, plasmid diversity, and streptomycin resistance. Genomics 112 : 3762-3772.

Singh, J., and Khan, A. 2019. Distinct patterns of natural selection determine subpopulation structure in the fire blight pathogen, Erwinia amylovora. Sci. Rep. 9: 14017.

Smits, T., Duffy, B., Sundin, G., Zhao, Y., and Rezzonico, F. 2017. Erwinia amylovora in the genomics era: From genomes to pathogen virulence, regulation, and disease control strategies. J. Plant Pathol. 99:7-23.

Smits, T. H., Rezzonico, F., Kamber, T., Blom, J., Goesmann, A., Frey, J. E., and Duffy, B. 2010. Complete genome sequence of the fire blight pathogen Erwinia amylovora CFBP 1430 and comparison to other Erwinia spp. Mol. Plant-Microbe Interact 23:384-393.

Smits, T. H. M. 2019. The importance of genome sequence quality to microbial comparative genomics. BMC Genomics 20:662.

Tatusova, T., DiCuccio, M., Badretdin, A., Chetvernin, V., Nawrocki, E. P., Zaslavsky, L., Lomsadze, A., Pruitt, K. D., Borodovsky, M., and Ostell, J. 2016. NCBI prokaryotic genome annotation pipeline. Nucleic Acids Res. 44:6614-6624.

van der Zwet, T. 1979. Fire blight: A bacterial disease of rosaceous plants. United States Department of Agriculture, Science and Education Administration, Washington, D.C.

Walker, B. J., Abeel, T., Shea, T., Priest, M., Abouelliel, A., Sakthikumar, S., Cuomo, C. A., Zeng, Q., Wortman, J., Young, S. K., and Earl, A. M. 2014. Pilon: An integrated tool for comprehensive microbial variant detection and genome assembly improvement. PLoS One 9:e112963.

Wang, D., Korban, S. S., and Zhao, Y. 2010. Molecular signature of differential virulence in natural isolates of Erwinia amylovora. Phytopathology 100:192-198.

Wick, R. R., Judd, L. M., Gorrie, C. L., and Holt, K. E. 2017. Unicycler: Resolving bacterial genome assemblies from short and long sequencing reads. PLOS Comput. Biol. 13:e1005595.

Wick, R. R., Judd, L. M., and Holt, K. E. 2019. Performance of neural network basecalling tools for Oxford Nanopore sequencing. Genome Biol. 20:129.

Yang, F., Korban, S. S., Pusey, P. L., Elofsson, M., Sundin, G. W., and Zhao, Y. 2014. Small-molecule inhibitors suppress the expression of both type III secretion and amylovoran biosynthesis genes in Erwinia amylovora. Mol. Plant Pathol. 15: 44-57.

Zeng, Q., Cui, Z., Wang, J., Childs, K. L., Sundin, G. W., Cooley, D. R., Yang, C. H., Garofalo, E., Eaton, A., Huntley, R. B., Yuan, X., and Schultes, N. P. 2018. Comparative genomics of Spiraeoideae-infecting Erwinia amylovora strains provides novel insight to genetic diversity and identifies the genetic basis of a low-virulence strain. Mol. Plant Pathol. 19:1652-1666.

Zhao, Y. 2014. Genomics of Erwinia amylovora and related Erwinia species associated with pome fruit trees. Pages 1-36 in: Genomics of Plant-Associated Bacteria. Springer, Berlin.

Zhao, Y., Blumer, S. E., and Sundin, G. W. 2005. Identification of Erwinia amylovora genes induced during infection of immature pear tissue. J. Bacteriol. 187: 8088-8103.

Zhao, Y., and Qi, M. 2011. Comparative genomics of Erwinia amylovora and related Erwinia species-What do we learn? Genes (Basel) 2:627-639. 